\begin{tabular}{|c|c|}
\hline Title & Three dimensional symmetry-breaking nontrivial topological states \\
\hline Author(s) & Habe, Tetsuro; A sano, Y asuhiro \\
\hline Citation & $\begin{array}{l}\text { Physical Review B, 89(11), 115203-1-115203-5 } \\
\text { https://doi.org/10.1103/PhysRevB.89.115203 }\end{array}$ \\
\hline Issue Date & $2014-03-20$ \\
\hline Doc URL & http:/hdl.handle.net/2115/56391 \\
\hline Rights & @2014 A merican Physical Society \\
\hline Type & article \\
\hline File Information & Phy sRevB89-11 115203.pdf \\
\hline
\end{tabular}

Instructions for use 


\title{
Three-dimensional symmetry-breaking nontrivial topological states
}

\author{
Tetsuro Habe ${ }^{1}$ and Yasuhiro Asano ${ }^{1,2}$ \\ ${ }^{1}$ Department of Applied Physics, Hokkaido University, Sapporo 060-8628, Japan \\ ${ }^{2}$ Center for Topological Science \& Technology, Hokkaido University, Sapporo 060-8628, Japan \\ (Received 19 July 2013; revised manuscript received 3 February 2014; published 20 March 2014)
}

\begin{abstract}
We discuss topological electronic states described by the Dirac Hamiltonian plus an additional one in three dimensions. When the additional Hamiltonian is an element of an Abelian group, electronic states become topologically nontrivial even in the absence of the fundamental symmetries such as the time-reversal symmetry and the particle-hole one. Such symmetry-breaking topological states are characterized by the Chern number defined in the two-dimensional partial Brillouin zone. The topological insulators in Zeeman fields are an example of the symmetry-breaking topological electric state. We show the crossover from the topological insulating phase to the topological semimetal one in strong Zeeman fields.
\end{abstract}

DOI: 10.1103/PhysRevB.89.115203

PACS number(s): 73.20.At, 73.20.Hb

Topological classification has successfully predicted a number of topologically nontrivial electronic states in the condensed matter. Each topological phase is characterized by a topological number defined in the presence of the fundamental symmetries preserved in the materials such as the time-reversal symmetry in topological insulators [1-4], the particle-hole symmetry in superfluids and topological superconductors [5,6], and the crystal symmetry in topological crystalline insulators $[7,8]$. The table of the topological classes $[9,10]$ has suggested a close relationship between the appearance of the topological phase and the invariance of the Hamiltonian under the fundamental symmetries. However, the surface states, an evidence of the topological phase, often remain gapless even when the fundamental symmetries are broken by perturbations. For instance, $Z_{2}$ number in the presence of the time-reversal symmetry characterizes the topological state of the ${ }^{3} \mathrm{He}-\mathrm{B}$ phase belonging to class DIII. In the Zeeman field, however, ${ }^{3} \mathrm{He}-\mathrm{B}$ phase still hosts the gapless states on a surface parallel to the Zeeman field. Mizushima et al. [11] have explained the existence of the gapless surface states in terms of a topological number defined by using the remaining symmetry of the ${ }^{3} \mathrm{He}-\mathrm{B}$ phase under the Zeeman field. Finding a particular topological number for explaining a particular gapless surface states is a way to understand physics of topological materials. However, there may be a more general route to characterize topological electronic states.

In this paper, we will show an alternative way to search the topological materials in three dimensions in the absence of the fundamental symmetries. The electronic structures of topological materials are described by the $4 \times 4$ Dirac Hamiltonian. A unitary transformation in the two-dimensional partial Brillouin zone (BZ) deforms the $4 \times 4$ Dirac Hamiltonian into two decoupled $2 \times 2$ quantum Hall Hamiltonians whose electric states are characterized by the nontrivial Chern number. The Chern number characterizes the two-dimensional topological states in class A where the Hamiltonian is not necessary to preserve any fundamental symmetries. Therefore the Chern number in each quantum Hall state (QHS) remains unchanged as far as perturbations do not mix them. Such perturbed Hamiltonian preserves a certain symmetry in the two-dimensional BZ but is not necessary to hold the fundamental symmetries globally in all the whole BZ. Therefore, the Dirac Hamiltonian plus such perturbed Hamiltonian describes topological states characterized by the Chern number without any fundamental symmetries. Our method to define the topological states in terms of the symmetry held locally in the partial BZ can be a tool to search topologically nontrivial states in novel materials. On the basis of the prescription, we propose semimetal phases of a time-reversal invariant topological insulator under the strong Zeeman field. Such semimetals are an example of the symmetry-breaking topological materials because they are characterized only by the Chern number in the partial BZ.

We begin with the three-dimensional Dirac Hamiltonian which describes electronic structures of topological materials such as topological insulators, topological superconductors, and superfluids,

$$
\begin{gathered}
H_{0}=a \alpha^{\mu} p_{\mu}+M \beta, \quad \mu=x, y, z, \\
M=\left(m-b p^{2}\right),
\end{gathered}
$$

where $a, b$, and $m$ are positive constants, $\alpha$ and $\beta$ are the $4 \times 4$ Dirac matrices,

$$
\alpha^{\mu}=\sigma^{\mu} \tau^{x}=\left(\begin{array}{cc}
0 & \sigma^{\mu} \\
\sigma^{\mu} & 0
\end{array}\right), \quad \beta=\sigma^{0} \tau^{z} .
$$

The diagonal $2 \times 2$ blocked sectors describe the two orbital spaces in the topological insulators [12] or the particle-hole subspace in the topological superconductors. Here the Pauli matrices $\sigma^{\mu}$ and $\tau^{\mu}$ for $\mu=x, y, z$ act on the spin and the orbital indices of an electron, respectively. The matrices $\sigma^{0}$ and $\tau^{0}$ are the $2 \times 2$ identity matrix in the corresponding subspace. The index which appears twice in a single term means the summation for $\mu=x, y, z$.

We first focus on a partial Brillouin zone specified by $p_{z}=0$. The Hamiltonian can be separated into two blocks as

$$
\begin{gathered}
H_{0}^{U}=\left(\begin{array}{cc}
\boldsymbol{d}(M) \cdot \boldsymbol{\sigma} & 0 \\
0 & \boldsymbol{d}(-M) \cdot \boldsymbol{\sigma}
\end{array}\right), \\
\boldsymbol{d}(M)=\left(a p_{x}, a p_{y}, M_{p_{z}=0}\right),
\end{gathered}
$$




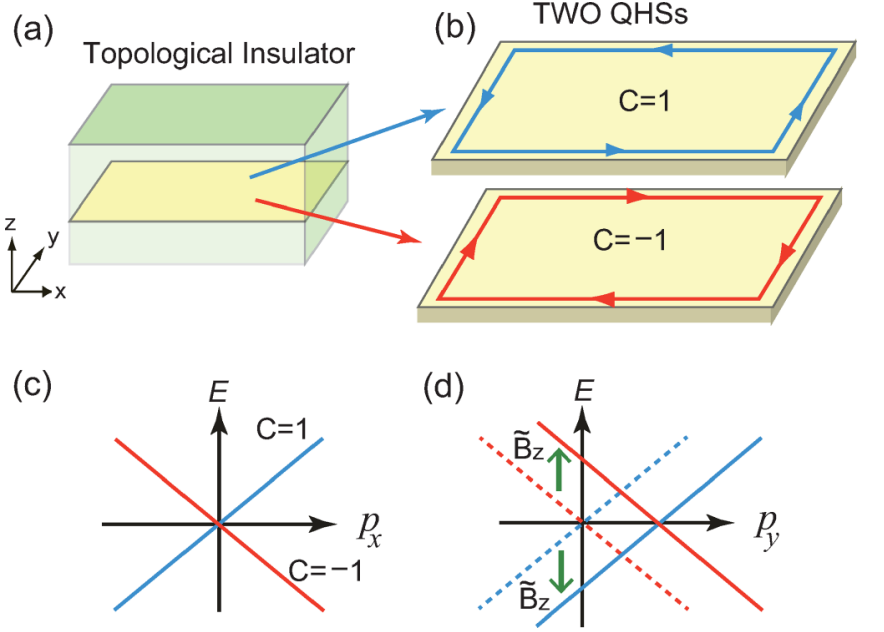

FIG. 1. (Color online) In (a), a plane represents the twodimensional partial Brillouin zone (BZ) embedded in the threedimensional one. In the partial BZ, the two blocked Hamiltonians $h(M)$ and $h(-M)$ describe the two quantum Hall states (QHSs) with the opposite chiral edge mode as shown in (b), where the arrows denote the direction of the chiral edge current. The corresponding dispersion of the edge modes is shown in (c). (d) Illustrates the dispersion of the edge modes under the antisymmetric Zeeman field which shifts the chemical potential of the two QHSs inversely. As a consequence, the Dirac point moves to another point in the BZ.

by applying the unitary transformation $H^{U}=U H U^{\dagger}$ with

$$
U=\left(\begin{array}{llll}
1 & 0 & 0 & 0 \\
0 & 0 & 0 & 1 \\
0 & 0 & 1 & 0 \\
0 & 1 & 0 & 0
\end{array}\right) .
$$

The Hamiltonian of the blocked sector $h( \pm M)=\boldsymbol{d}( \pm M) \cdot \sigma$ is a simple model of the quantum Hall systems [13] as shown in Fig. 1(b). The chiral edge modes of the two quantum Hall states (QHSs) have the opposite chirality to each other. When the Chern number $\mathrm{C}$ is 1 for $h(M)$, it is -1 for $h(-M)$. The dispersion of the chiral edge modes goes across the zero energy at the so-called Dirac point in $p_{x}$ and $p_{y}$ axes on the $x z$ and $y z$ surfaces, respectively, as schematically shown in Fig. 1(c). The Dirac point is stable even when we add a perturbation $H_{\mathcal{P}}^{U}$ to $H_{0}^{U}$ as far as $H_{\mathcal{P}}^{U}$ is invariant under the unitary transformation given by $\mathcal{P}_{x y}^{U}=\tau^{z}$ [i.e., $\left.\mathcal{P}_{x y}^{U} H_{\mathcal{P}}^{U}\left(\mathcal{P}_{x y}^{U}\right)^{\dagger}=H_{\mathcal{P}}^{U}\right]$. This is because such $H_{\mathcal{P}}^{U}$ does not mix the two QHSs. In the original representation before applying $U$, the perturbed Hamiltonian $H_{\mathcal{P}}$ should satisfy $\mathcal{P}_{x y} H_{\mathcal{P}} \mathcal{P}_{x y}^{\dagger}=$ $H_{\mathcal{P}}$, where $\mathcal{P}_{x y}=\sigma^{z} \tau^{z}$ represents the combined operation of the pseudospin inversion $\tau^{\mu} \rightarrow-\tau^{\mu}$ and spin inversion $\sigma^{\mu} \rightarrow-\sigma^{\mu}$ for $\mu=x$ and $y$. The set of Hermite matrices $H_{\mathcal{P}}$ forms an Abelian group $A_{P}$ whose elements are invariant under the unitary transformation by $\mathcal{P}_{x y}$. Nontrivial Chern numbers in the partial $\mathrm{BZ}$ of the $p_{x} p_{y}$ plane explain the existence of the gapless states on the four surfaces perpendicular to the $x y$ plane. It is possible to generalize the above argument to the partial BZ specified by $p_{\rho}=0$. In this case, the unitary operator is represented by

$$
\mathcal{P}_{\mu \nu}=\epsilon_{\mu \nu \rho} \sigma^{\rho} \tau^{z},
$$

where $\epsilon_{\mu \nu \rho}$ is antisymmetric symbol. Using the property of the Abelian group is a simple way to describe physics of the present issue. But it is not essential. To advance concrete discussions on $H_{\mathcal{P}}$, we explicitly express an example of $H_{\mathcal{P}}$,

$$
\left(\begin{array}{cc}
a_{0}+a_{\rho} \sigma^{\rho} & b_{\mu} \sigma^{\mu}+b_{\nu} \sigma^{\nu} \\
b_{\mu}^{*} \sigma^{\mu}+b_{\nu}^{*} \sigma^{\nu} & c_{0}+c_{\rho} \sigma^{\rho}
\end{array}\right),
$$

where $\mu, v$, and $\rho$ represent $x, y$, and $z$, and they are not equal to one another. Coefficients $a_{0}, a_{\rho}, c_{0}$, and $c_{\rho}$ are real numbers but they are not necessary to be constants. The additional Hamiltonian contains the various effects: the intraband scattering without spin flip for $a_{0}$ and $c_{0}$, that with spin flip for $a_{\mu}$ and $c_{\mu}$, the modulation of spin-orbit interaction, e.g., anisotropy, for $b_{\mu}$, and etc.

The Abelian group $A_{P}$ has a subgroup of $A_{P Q}$. The perturbed Hamiltonian belonging to $A_{P Q}$ does not remove the gapless states on all the surfaces. To show this property, we consider the elements that commute with $\mathcal{P}_{y z}$. The gapless states remain on the four surfaces perpendicular to the $y z$ plane under such perturbed Hamiltonian. Thus we show the gapless states on the remaining two surfaces parallel to the $y z$ plane. After applying the transformation by $U$ in Eq. (5), Eq. (1) with $p_{y}=p_{z}=0$ becomes

$$
\begin{aligned}
H_{0}^{W U} & =W H_{0}^{U} W^{\dagger}=\left(\begin{array}{cc}
\boldsymbol{d}^{\prime}(M) \cdot \boldsymbol{\sigma} & 0 \\
0 & \boldsymbol{d}^{\prime}(M) \cdot \boldsymbol{\sigma}
\end{array}\right), \\
\boldsymbol{d}^{\prime}(M) & =\left(a p_{x}, 0, m-b p_{x}^{2}\right),
\end{aligned}
$$

where $W$ is an unitary matrix of $W=\operatorname{diag}\left[\sigma^{0},-i \sigma^{x}\right]$. At the point of $p_{y}=p_{z}=0$, the edge states on the $y z$ surface are degenerate at the zero energy. This is because the two blocked sectors are equivalent to each other in this representation and because each blocked sector preserves the "particle-hole symmetry" within the $2 \times 2$ space represented by a relation $\sigma^{y}\left(\boldsymbol{d}^{\prime} \cdot \boldsymbol{\sigma}\right) \sigma^{y}=-\boldsymbol{d}^{\prime} \cdot \boldsymbol{\sigma}$. Thus the degeneracy of the two edge states remains even when we add the "particle-hole" symmetrical Hamiltonian $H_{\mathcal{Q}}^{W U}$ to $H_{0}^{W U}$. Such Hamiltonian $H_{\mathcal{Q}}^{W U}$ should satisfy the relation $\mathcal{Q}_{y z}^{W U} H_{\mathcal{Q}}^{W U}\left(\mathcal{Q}_{y z}^{W U}\right)^{\dagger}=-H_{\mathcal{Q}}^{W U}$ with $\mathcal{Q}_{y z}^{W U}=\sigma^{y} \tau^{0}$. The Hermitian matrices form the Abelian subgroup $A_{P Q}$ whose elements $H_{\mathcal{P} \mathcal{Q}}$ satisfy $\mathcal{Q}_{y z} H_{\mathcal{P} \mathcal{Q}} \mathcal{Q}_{y z}^{\dagger}=$ $-H_{\mathcal{P} \mathcal{Q}}$ and $\mathcal{P}_{y z} H_{\mathcal{P} \mathcal{Q}} \mathcal{P}_{y z}^{\dagger}=H_{\mathcal{P} \mathcal{Q}}$ at the same time. In the original representation before applying the transformation of $W$ and $U$, the unitary matrix $\mathcal{Q}_{y z}$ is given by

$$
\mathcal{Q}_{y z}=\sigma^{x} \tau^{y} \text {. }
$$

When we consider elements invariant under $\mathcal{P}_{\mu \nu}$ in general, the unitary matrix in Eq. (9) is represented as $\mathcal{Q}_{\mu \nu}=\epsilon_{\mu \nu \rho} \sigma^{\rho} \tau^{y}$. In addition to the elements in $A_{P Q}$, the Hamiltonian proportional to the $4 \times 4$ identity matrix does not affect the gapless surface states at all.

In a short summary, we have discussed the threedimensional symmetry-breaking topological state whose electronic structures are represented by

$$
H=H_{0}+H_{\mathcal{P}},
$$


where $H_{\mathcal{P}}$ commutes with $\mathcal{P}_{\mu \nu}=\epsilon_{\mu \nu \rho} \sigma^{\rho} \tau^{z}$. The set of such Hamiltonian forms the Abelian group $A_{\mathcal{P}}$. The Chern number defined in the two-dimensional partial BZ specified by $p_{\rho}=$ 0 characterizes the topologically nontrivial states. Generally speaking, the symmetry in a two-dimensional partial BZ is not necessary to be held in whole BZ in three dimensions. In addition, the gapped energy spectra in a two-dimensional partial BZ are not necessary to be held in whole BZ in three dimensions. The word "symmetry breaking" means that the symmetries preserved in $H_{0}$ are broken by $H_{\mathcal{P}}$, and those preserved in $H_{\mathcal{P}}$ are broken by $H_{0}$. In what follows, we discuss electronic states of the three-dimensional topological insulator under the Zeeman field as an example of the three-dimensional symmetry-breaking topological states.

The Zeeman field is represented by two vectors $\boldsymbol{B}$ and $\tilde{\boldsymbol{B}}$ as

$$
H_{1}=B_{\mu} \sigma^{\mu} \tau^{0}+\tilde{B}_{\mu} \sigma^{\mu} \tau^{z},
$$

where $B_{\mu} \sigma^{\mu} \tau^{0}$ and $\tilde{B}_{\mu} \sigma^{\mu} \tau^{z}$ are the symmetric and the antisymmetric parts of the Zeeman field with respect to the two orbitals, respectively. The antisymmetric part of $\tilde{B}_{\mu} \sigma^{\mu} \tau^{z}$ is attributed to the difference of the coupling constants to the Zeeman field in the two orbitals and is dominant in $\mathrm{Bi}_{2} \mathrm{Se}_{3}$ [12]. We consider the subgap states on the $y z$ surface for a while. When the weak Zeeman field of $B, \tilde{B}<m$ is applied along the $z$ axis parallel to the $y z$ surface, the two QHSs in the two-dimensional partial $\mathrm{BZ}$ on the $p_{y} p_{z}$ plane still remain decoupled from each other because the $H_{1}$ commutes with $\mathcal{P}_{x y}$. Applying the unitary transformation of $U$, the Hamiltonian becomes

$$
H_{1}^{U}=B_{z} \sigma^{z} \tau^{0}+\tilde{B}_{z} \sigma^{0} \tau^{z} .
$$

The symmetric Zeeman field $B_{z}$ gives a constant correction to $M$ in Eq. (3) and does not affect the Dirac point in twodimensional $\mathrm{BZ}$ at all. On the other hand, the antisymmetric Zeeman field $\tilde{B}_{z}$ shifts the chemical potential of the two QHSs inversely. As a result, the Dirac point moves from the $\Gamma$ point $\left(p_{y}, p_{z}\right)=0$ as shown in Fig. 1(d). The situation is similar to the shift of the Dirac point at the interface facing to a ferromagnetic insulator [14-17].

Next we consider the Zeeman field in the direction perpendicular to the $y z$ surface. We conclude that the symmetric Zeeman field would remove the gapless states from the $y z$ surface because it does not belong to the Abelian subgroup $A_{P Q}$. On the other hand, the antisymmetric Zeeman field leaves the gapless states because it belongs to the Abelian subgroup $A_{P Q}$. It is easy to confirm these conclusions by the argument below. Both the symmetric $B_{x} \sigma^{x} \tau^{0}$ and the antisymmetric $\tilde{B}_{x} \sigma^{x} \tau^{z}$ Zeeman field commute with $\mathcal{P}_{y z}=$ $\sigma^{x} \tau^{z}$ but do not commute with either $\mathcal{P}_{x y}=\sigma^{z} \tau^{z}$ or $\mathcal{P}_{x z}=$ $-\sigma^{y} \tau^{z}$. The symmetric Zeeman field does not anticommute with $Q_{y z}$ in Eq. (9), which indicates the gapless states are no longer guaranteed on the $y z$ surface. On the other hand, the antisymmetric Zeeman field anticommutes with $Q_{y z}$, which means the antisymmetric Zeeman field belongs to $A_{P Q}$. This is because the antisymmetric Zeeman field preserves the "particle-hole symmetry" in each QHS. As a result, the symmetric (antisymmetric) Zeeman field removes (leaves) the gapless energy spectra on the surface perpendicular to the Zeeman field. So far we have considered the weak Zeeman field of $m>|B|$ and $m>|\tilde{B}|$. In the strong Zeeman field
$m<|B|$ and $m<|\tilde{B}|$, another topological phases would be also expected because the two QHSs still remain decoupled from each other. We will confirm the last statement by numerical calculation.

In what follows, we confirm the analysis above by the numerical calculation for the combined Hamiltonian $H=$ $H_{0}+H_{1}$ on the tight-binding lattice,

$$
\begin{aligned}
H= & \sum_{\boldsymbol{p}} \boldsymbol{c}(\boldsymbol{p})^{\dagger}\left[\left(m-b \sum_{\nu=x, y, z}\left(1-\cos p_{\nu}\right)\right) \sigma^{0} \tau^{z}\right. \\
& \left.+a \sin p_{\mu} \sigma^{\mu} \tau^{x}+\sigma^{\nu}\left(B_{v} \tau^{0}+\tilde{B}_{\nu} \tau^{z}\right)\right] \boldsymbol{c}(\boldsymbol{p}),
\end{aligned}
$$

with the parameters $m, a$, and $b$ used in Ref. [17]. Here $\boldsymbol{c}(\boldsymbol{p})=\left(c_{1 \uparrow}, c_{1 \downarrow}, c_{2 \uparrow}, c_{2 \downarrow}\right)^{T}$ is the annihilation operator with four components corresponding to spin $\uparrow, \downarrow$ and orbital 1,2 subspaces. To calculate the energy spectra of the surface state on the $y z$ plane, we consider the lattice along $x$ axis with

$$
\begin{aligned}
\sum_{p_{x}} \cos p_{x} c^{\dagger}\left(p_{x}\right) c\left(p_{x}\right) & \rightarrow \frac{1}{2} \sum_{j}\left(c^{\dagger}(j+1) c(j)+\text { H.c. }\right), \\
\sum_{p_{x}} \sin p_{x} c^{\dagger}\left(p_{x}\right) c\left(p_{x}\right) & \rightarrow \frac{1}{2 i} \sum_{j}\left(c^{\dagger}(j+1) c(j)-\text { H.c. }\right),
\end{aligned}
$$

where we utilize $j$ as the position on the $x$ axis and employee the hard-wall boundary condition in the $x$ axis.

At first, we consider the weak Zeeman field. The topological phases can be confirmed by the appearance of the gapless surface states. In Fig. 2, we show the energy spectra of the surface states on the $y z$ plane under the symmetric Zeeman field with $|B|=m / 2$ and $|\tilde{B}|=0$ in (a) and (c), and those
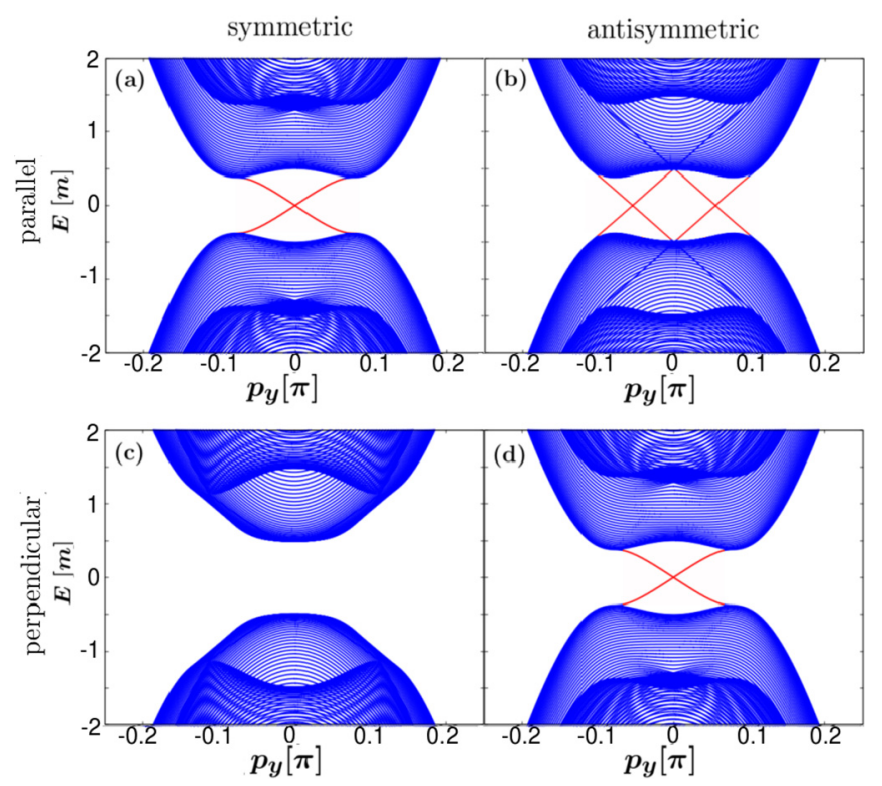

FIG. 2. (Color online) The energy spectra of the $y z$ surface states under the symmetric Zeeman field (a) and (c) or the antisymmetric Zeeman field (b) and (d). The Zeeman field is applied in a parallel direction to the surface in (a) and (b). In (c) and (d), the Zeeman field is applied in the perpendicular direction to the surface. The momentum in the $z$ direction is fixed at $p_{z}=0$ in all figures. 

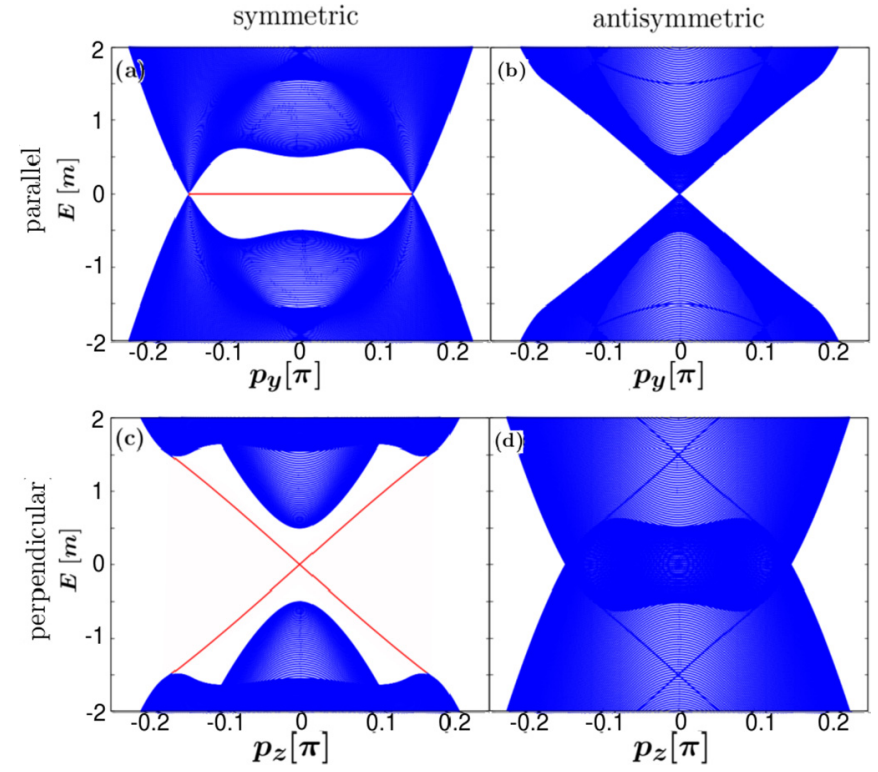

FIG. 3. (Color online) The energy spectra on the $y z$ surface under the large Zeeman field applied in the $y$ direction. The results are plotted along the $p_{y}$ axis with $p_{z}=0$ (upper figures) and along the $p_{z}$ axis with $p_{y}=0$ (lower figures). The left figures of (a) and (c) are the results for the symmetric Zeeman field at $B_{y}=1.5 \mathrm{~m}$. The right figures of (b) and (d) are calculated under the antisymmetric Zeeman field at $\tilde{B}_{y}=1.5 \mathrm{~m}$.

under the antisymmetric Zeeman field $|B|=0$ and $|\tilde{B}|=m / 2$ in (b) and (d). The results for the Zeeman field parallel to the $y z$ surface are shown in Figs. 2(a) and 2(b). The energy spectra on the surface remain gapless in both the symmetric and the antisymmetric Zeeman field. Especially, under the antisymmetric field in Fig. 2(b), the Dirac point shifts from the $\Gamma$ point. When the symmetric Zeeman field is perpendicular to the surface, the gapless states on the $y z$ plane vanish as shown in Fig. 2(c). On the other hand, as shown in Fig. 2(d), the gapless states survive under the antisymmetric Zeeman field. The numerical results in Fig. 2 suggest the validity of the analysis.

Finally, we show the numerical results for the large Zeeman field $m<|B|$ and $m<|\tilde{B}|$. According to Eqs. (3) and (12), the large enough symmetric Zeeman field equalizes two of the Chern numbers of the two QHSs because $\operatorname{sgn}[M+B]=$ $\operatorname{sgn}[-M+B]$. Therefore, the net Chern number becomes nontrivial in the two-dimensional BZ. The electronic states of the topological insulator under the large symmetric Zeeman field consist of a number of QHSs with the same Chern number stacking in momentum space. As a consequence, electronic states become the Weyl semimetal phase [18-21].
In Figs. 3(a) and 3(c), we show the energy spectra on the $y z$ surface under the strong symmetric Zeeman field in the $y$ axis with $B_{y}=1.5 \mathrm{~m}$. The semimetal hosts the chiral surface modes, so-called the Fermi arc as shown by a pair of linear dispersion in (c). The spectra in Fig. 3 include the contribution from the two $y z$ planes parallel to the Zeeman field. When one $y z$ surface hosts a chiral mode with the positive velocity, the other $y z$ surface hosts a chiral mode with the negative velocity. The results are consistent with a study of the large Zeeman field in Ref. [22]. In the purely two-dimensional system, the semimetallic states are realized in the junction between a TI and a ferromagnetic insulator [23]. On the other hand, the antisymmetric Zeeman field biases the two QHSs reversely but holds the Chern number unchanged. As a result, the topological insulator qualitatively changes into the nodal semimetal as shown in Figs. 3(b) and 3(d), where we show the excitation spectra of bulk states under the strong antisymmetric Zeeman field in the $y$ axis with $\tilde{B}_{y}=1.5 \mathrm{~m}$. We have confirmed that the semimetallic states always appear even when the two types of Zeeman components coexist. The characteristic feature of the resulting semimetallic state is dominated by the larger component. The numerical results in Fig. 3 might imply the applicability of our approach to metallic materials.

In summary, we have discussed topologically nontrivial electronic states described by the Hamiltonian of $H=H_{0}+$ $H_{\mathcal{P}}$, where $H_{0}$ is the Dirac Hamiltonian, and $H_{\mathcal{P}}$ is an additional Hamiltonian belonging to an Abelian group of $A_{P}$. The Chern number defined in the two-dimensional partial Brillouin zone characterizes the topological states. Generally speaking, such topological states can be realized without any fundamental symmetries such as the time-reversal, the particlehole, and the chiral symmetries. Thus we have proposed a possibility of a symmetry-breaking topological state in three dimensions. When $H_{\mathcal{P}}$ belongs to an Abelian subgroup of $A_{P Q}$, the symmetry-breaking topological state has the gapless states on all the surfaces. We have numerically studied the electronic states in a topological insulator in Zeeman fields as an example of the three-dimensional symmetry-breaking topological states. The results of the low-energy excitation spectra suggest the Weyl semimetal phase or the line-nodal semimetal one depending on characters of large Zeeman fields. In some topological insulators, the $m$ is controllable in the range of $0-500 \mathrm{meV}[24,25]$. Therefore it would be possible to confirm the proposed semimetal phase in experiments.

The authors are grateful to K. Nomura for useful comments on our manuscript. This work was supported by the "Topological Quantum Phenomena" (Grant No. 22103002) Grant-in Aid for Scientific Research on Innovative Areas from the Ministry of Education, Culture, Sports, Science and Technology (MEXT) of Japan.
[1] L. Fu, C. L. Kane, and E. J. Mele, Phys. Rev. Lett. 98, 106803 (2007).

[2] L. Fu and C. L. Kane, Phys. Rev. B 76, 045302 (2007).

[3] J. E. Moore and L. Balents, Phys. Rev. B 75, 121306 (2007).
[4] Y. L. Chen, J. G. Analytis, J.-H. Chu, Z. K. Liu, S. K. Mo, X. L. Qi, H. J. Zhang, D. H. Lu, X. Dai, Z. Fang, S. C. Zhang, I. R. Fisher, Z. Hussain, and Z. X. Shen, Science 325, 178 (2009). 
[5] X.-L. Qi, T. L. Hughes, S. Raghu, and S.-C. Zhang, Phys. Rev. Lett. 102, 187001 (2009).

[6] L. Fu and E. Berg, Phys. Rev. Lett. 105, 097001 (2010).

[7] L. Fu, Phys. Rev. Lett. 106, 106802 (2011).

[8] T. H. Hsieh, H. Lin, J. Liu, W. Duan, A. Bansil, and L. Fu, Nat. Comm. 3, 982 (2012).

[9] A. P. Schnyder, S. Ryu, A. Furusaki, and A. W. W. Ludwig, Phys. Rev. B 78, 195125 (2008).

[10] C.-K. Chiu, H. Yao, and S. Ryu, Phys. Rev. B 88, 075142 (2013).

[11] T. Mizushima, M. Sato, and K. Machida, Phys. Rev. Lett. 109, 165301 (2012).

[12] C.-X. Liu, X.-L. Qi, H. J. Zhang, X. Dai, Z. Fang, and S.-C. Zhang, Phys. Rev. B 82, 045122 (2010).

[13] X.-L. Qi, T. L. Hughes, and S.-C. Zhang, Phys. Rev. B 78, 195424 (2008).

[14] Y. Tanaka, T. Yokoyama, and N. Nagaosa, Phys. Rev. Lett. 103, 107002 (2009).

[15] Q. Liu, C.-X. Liu, C. Xu, X.-L. Qi, and S.-C. Zhang, Phys. Rev. Lett. 102, 156603 (2009).
[16] L. A. Wray, S.-Y. Xu, Y. Xia, D. Hsieh, A. V. Fedorov, Y. S. Hor, R. J. Cava, A. Bansil, H. Lin, and M. Z. Hasan, Nat. Phys. 7, 32 (2010).

[17] T. Habe and Y. Asano, Phys. Rev. B 85, 195325 (2012).

[18] S. Murakami, New J. Phys. 9, 356 (2007).

[19] X. Wan, A. M. Turner, A. Vishwanath, and S. Y. Savrasov, Phys. Rev. B 83, 205101 (2011).

[20] K.-Y. Yang, Y.-M. Lu, and Y. Ran, Phys. Rev. B 84, 075129 (2011).

[21] A. A. Burkov and L. Balents, Phys. Rev. Lett. 107, 127205 (2011).

[22] C.-X. Liu, P. Ye, and X.-L. Qi, Phys. Rev. B 87, 235306 (2013).

[23] T. Paananen and T. Dahm, Phys. Rev. B 87, 195447 (2013).

[24] Z. Ren, A. A. Taskin, S. Sasaki, K. Segawa, and Y. Ando, Phys. Rev. B 84, 165311 (2011).

[25] S.-Y. Xu, Y. Xia, L. A. Wray, S. Jia, F. Meier, J. H. Dil, J. Osterwalder, B. Slomski, A. Bansil, H. Lin, R. J. Cava, and M. Z. Hasan, Science 332, 560 (2011). 